\title{
Estación Meteorológica para la Conformación de Redes. Proceso de Instalación
}

\author{
Meteorological Station for forming networks .Installation Process
}

\author{
German Arturo López Martínez \\ Facultad Tecnológica, Universidad Distrital, Bogotá, Colombia \\ germanlopezmeyahoo.es
}

\begin{abstract}
Resumen-A finales de 2014 se instala en la Facultad Tecnológica de la Universidad Distrital en Bogotá, Colombia una estación meteorológica para la medición y almacenamiento de valores promedios de variables meteorológicas en la localidad Ciudad Bolívar como radiación solar, precipitación, dirección y velocidad del viento, entre otras. Se utiliza, como hardware, un dispositivo inalámbrico de transmisión, automático y compacto marca PEGASUS.

Se enfatiza en el procedimiento para la escogencia del lugar más conveniente para el montaje de la estación, siguiendo las normas de la OMM (Organización Meteorológica Mundial), recomendaciones del IDEAM (Instituto de Hidrología, Meteorología y Estudios Ambientales de Colombia) y del fabricante. Se espera que la estación sea parte de una red de estaciones de algunas universidades de Bogotá pertenecientes a la Red Internacional para la Promoción de la Investigación y Docencia en Energización Rural para el desarrollo Sostenible (Red PRIDERAS), también ser parte de la red del IDEAM.).
\end{abstract}

Palabras clave- Meteorología, radiación solar, precipitación, velocidad y dirección del viento, redes.

Abstract- In late 2014 it installs, in the headquarters of the Faculty of Technology of the Universidad Distrital, in Bogota Colombia, a weather station for measuring and storing variables such as solar radiation, rainfall, direction and wind speed, among others, in order to have information to support research projects in the Universidad Distrital and other research centers.

The station hardware uses a wireless transmission device, it is automatic, compact, easy to install, and this allows to obtain average valuesof meteorological variables in the Localidad Ciudad Bolivar, Bogota. This document emphasizes the procedure for the selection of the most suitable for mounting the weather station place, following the rules of the WMO (World Meteorological Organization), the recommendations of the IDEAM (Instituto de Hidrologia, Meteorología y Estudios Ambientales de Colombia) and the manufacturer. It is expected that the station is part of a network of stations of some universities in Bogotá belonging to the International Network for the Promotion of Research and Teaching in Rural Energy for
Sustainable Development (Red PRIDERAS). Additionally it aims to be part of the network of meteorological stations IDEAM.

Key Word - Meteorology, solar radiation, precipitation, wind speed and direction, networks.

\section{INTRODUCCIÓN}

Estación Meteorológica es el lugar donde se hacen observaciones y mediciones puntuales de diferentes parámetros meteorológicos usando instrumentos apropiados, con el fin de establecer el comportamiento atmosférico en las diferentes zonas de un territorio. [1]

La instalación de cada estación y su integración a una red meteorológica permite representar los procesos ambientales de las sub zonas climatológicas del territorio nacional, cubrir las zonas de mayor importancia socioeconómica, superar la insuficiencia en cobertura de estaciones en determinadas áreas, conocer el comportamiento climático de los diferentes ecosistemas, ampliar la cobertura a zonas de interés nacional sin información meteorológica, el seguimiento de procesos biometeorológicos, como también representar los procesos ambientales de los principales conglomerados urbanos y áreas vulnerables a deslizamientos.(IDEAM)

La estación meteorológica instalada en la Facultad tecnológica es de tipo automática, está destinada a la toma de datos meteorológicos en el sur de Bogotá, lo que le permitirá a la Universidad Distrital contar con información, en tiempo real, del comportamiento de las variables meteorológicas en esta zona.

El grupo de investigación en energías alternativas de la Universidad Distrital (GIEA-UD), plantea este proyecto con el fin de recolectar y procesar datos meteorológicos y emitir reportes a la comunidad académica de la Universidad Distrital y a la sociedad bogotana, con el objetivo de establecer el potencial energético renovable en esta zona de la ciudad, dato esencial para servir de base en futuros proyectos tecnológicos conducentes a la explotación sostenible de estos recursos, y 
conformar, a futuro, una red de datos meteorológicos con otros centros de investigación y universidades de Bogotá.

\section{ESTACIÓN METEOROLÓGICA}

La estación tiene como características principales las siguientes: es inalámbrica, de fácil instalación, permite la obtención de datos meteorológicos mediante el uso de diversos sensores; suministra información adicional a partir del procesamiento de las mediciones, permite el registro y visualización de datos instantáneos e históricos y, tiene la opción de comunicarse con un computador personal, para almacenar los datos.

Está conformada por tres componentes principales: una unidad externa, una unidad de adquisición de datos y una consola.

La estación tiene como características principales las siguientes: es inalámbrica, de fácil instalación, permite la obtención de datos meteorológicos mediante el uso de diversos sensores; suministra información adicional a partir del procesamiento de las mediciones, permite el registro y visualización de datos instantáneos e históricos y, tiene la opción de comunicarse con un computador personal, para almacenar los datos.

Está conformada por tres componentes principales: una unidad externa, una unidad de adquisición de datos y una consola.

\section{A. Unidad externa.}

Unidad externa, que a su vez contiene la unidad de adquisición de datos (Figura 1), son las que realizan las mediciones por medio de sensores y las transmite mediante un enlace de radiofrecuencia a la consola; tiene un subsistema de alimentación de energía con panel solar y una batería de respaldo. Se montan sobre una estructura tipo trípode de altura variable. En ella se conectan los siguientes sensores: temperatura, humedad de ambiente exterior, velocidad y dirección del viento, precipitación y radiación solar; también pueden conectarse a la unidad de adquisición otros sensores como humedad de hoja, temperatura y humedad del suelo.

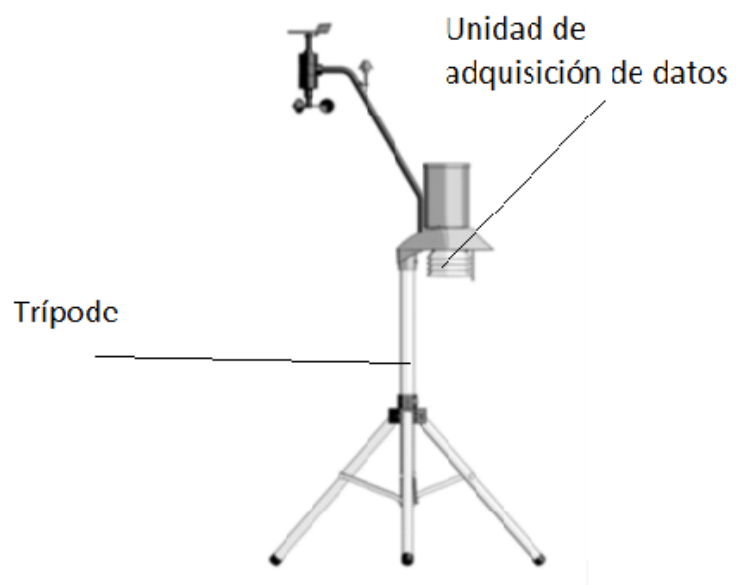

Fig. 1. Unidad externa con unidad de adquisición de datos. [2]
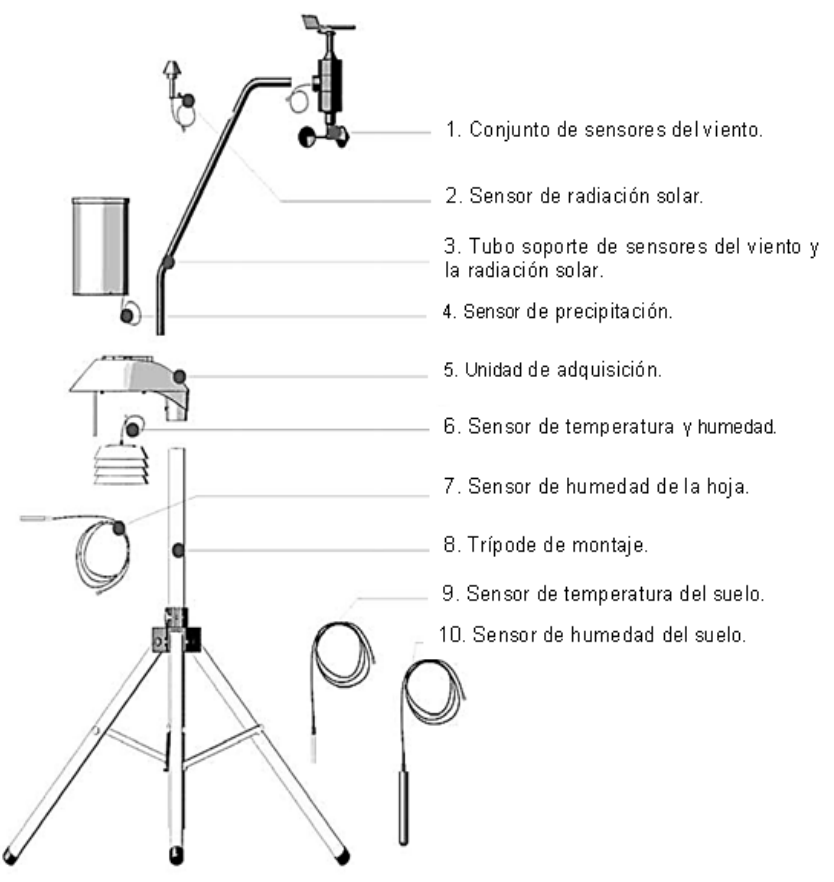

Fig. 2. Componentes de la unidad externa. [2]

\section{Características.}

La estación permite seleccionar el período de registro de los datos entre 5 y 60 minutos, tiene una capacidad de memoria de 14.000 datos por variable (equivalente a 145 días de autonomía con datos registrados cada 15 minutos, o 97 días con datos tomados cada 10 minutos). La unidad de adquisición de datos permite la conexión de nueve sensores, estos están relacionados en la Tabla 1, en ella se indican: el código del sensor, sus características, el rango de medición, la resolución y la exactitud, según la variable a medir. 


\begin{tabular}{|c|c|c|c|c|c|}
\hline MEDICIÓN & Sensor & Característica & RANGO & RESOLUCIÓN & EXACTITUD \\
\hline Precipitación & $\begin{array}{l}\text { Pluviómetro } \\
\text { EP0221 }\end{array}$ & $\begin{array}{l}\text { Boca de captación } \\
\text { metálica calibrada. }\end{array}$ & $0-300 \mathrm{~mm}$ & $0,25 \mathrm{~mm}$ & $\begin{array}{l}+/-4 \% \mathrm{a} \\
50 \mathrm{~mm} / \mathrm{h}\end{array}$ \\
\hline Velocidad del viento & EP0233: & $(1 \mathrm{~m} / \mathrm{s}$ a $50 \mathrm{~m} / \mathrm{s})$ & $0-200 \mathrm{~km} / \mathrm{h}$ & $0,1 \mathrm{~m} / \mathrm{s} ; 1 \mathrm{~km} / \mathrm{h}$ & $+/-5 \% \mathrm{FE}$ \\
\hline Dirección del viento & EP0233: & $\begin{array}{l}\text { Dirección de viento (de 0- } \\
360^{\circ} \text { ); }\end{array}$ & $0-3600$ & $22,5=$ & $+/-15 \%$ \\
\hline $\begin{array}{l}\text { Humedad del aire } \\
\text { exterior }\end{array}$ & EP0251 & & $0-100 \%$ & $1 \%$ & $+/-5 \%$ \\
\hline $\begin{array}{l}\text { Temperatura del } \\
\text { aire exterior }\end{array}$ & EP0251 & $\begin{array}{l}\text { Protector solar: de tipo } \\
\text { autoaspirante. }\end{array}$ & 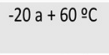 & $0,1 \stackrel{\circ}{ } \mathrm{C}$ & $+1-0,5 \stackrel{9}{C}$ \\
\hline Radiación solar & EP0304 & Tipo celda de silicio. & $1400 \mathrm{~W} / \mathrm{m}^{2}$ & $1 \mathrm{~W} / \mathrm{m}^{2}$ & $+/-5 \% \mathrm{FE}$ \\
\hline Humedad del suelo & EP0254 & $\begin{array}{l}\text { De tipo por constante } \\
\text { dieléctrica de alta durabilidad } \\
\text { que no requiere } \\
\text { mantenimiento. }\end{array}$ & $0-100 \%$ & $1 \%$ & $+/-5 \%$ \\
\hline $\begin{array}{l}\text { Temperatura del } \\
\text { suelo }\end{array}$ & EP0247 & & $-20 \mathrm{a}+60^{\circ} \mathrm{C}$ & $0,1^{\circ} \mathrm{C}$ & $+1-0,5^{\circ} \mathrm{C}$ \\
\hline Humedad de hoja & EP0253 & $\begin{array}{l}\text { sensor de hoja mojada } \\
\text { con accesorio de } \\
\text { instalación }\end{array}$ & N/A & N/A & N/A \\
\hline
\end{tabular}

Tabla 1. Sensores que se pueden conectar a la unidad de adquisición de datos.[2]

En la Fig. 2 se muestra los componentes y la disposición de los sensores en la unidad externa y de adquisición de datos.

\section{Unidad de adquisición.}

También conocida como adquisidor, a él se conectan todos los sensores, se encarga de recolectar los datos censados y de transmitir esta información a la consola.

El Sistema de montaje para la instalación del adquisidor, se hace sobre un tubo de $45 \mathrm{~mm}$ de diámetro ubicado sobre el trípode, funciona de manera autónoma usando una batería eléctrica que se carga con un panel solar, además calcula otras variables como: la velocidad y dirección de ráfagas del viento, y censa: la temperatura exterior, la humedad relativa del aire externo, la presión atmosférica, la humedad del suelo y la carga de la batería.

\section{B. Unidad interna o consola}

Es un equipo de mano, compacto, (Figura 3), tiene una pantalla gráfica de alta resolución, sensible al tacto, se usa en espacios interiores, implementa el registro de datos, enviados por la unidad de adquisición de datos $\mathrm{y}$, permite la visualización de valores instantáneos y curvas de tendencia generadas a partir de datos históricos. Posee un menú de configuración, mediante el cual se programan las condiciones de uso. La recepción de los datos se realiza a través de un enlace de radiofrecuencia.

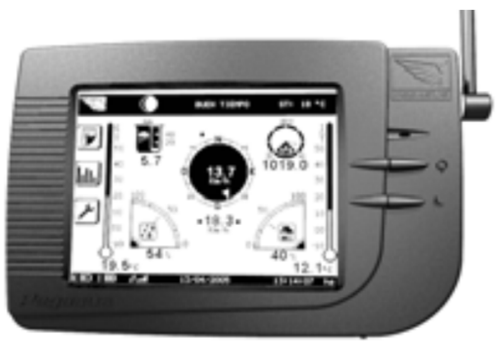

Fig. 3. Unidad interna o consola.[2]

\section{Características}

La consola de visualización es un dispositivo indicador con pantalla táctil de 120 x $92 \mathrm{~mm}$, permite el registro y visualización gráfica de los datos medidos y trasmitidos por la unidad adquisidora. La alimentación es a 100-240 Vca, cuenta con la opción de apagado/encendido, su pantalla de visualización es de cristal líquido con luz interior, el cambio de las modalidades de funcionamiento es del tipo contacto. Dispone de un puerto USB para conexión a un PC; posee 4 pantallas diferentes de: visualización gráfica de las variables, visualización digital, graficación y configuración.

Asimismo, permite censar la temperatura y humedad interna, y calcular y mostrar la precipitación en el período de medición, la dirección del viento y su velocidad en forma numérica y, en rosa de los vientos, la dirección y velocidad de las ráfagas de viento, el estado de carga de la batería del adquisidor, el estado de carga de la batería de la consola, los registros de: precipitación, humedad de hoja, punto de rocío, radiación solar diaria; además, presenta gráficos temporales acumulados en períodos de 2 y 7 días por cada variable.

\section{ESCOGENCIA DEL SITIO DE UBICACIÓN}

La sede de la Facultad Tecnológica de la Universidad Distrital Francisco José de Caldas está ubicada en la Localidad 19 de la ciudad de Bogotá, en las coordenadas: 4.5790626, Latitud Norte, y -74.1577043, Longitud Oeste. En la Figura 4 se aprecia una fotografía satelital de la sede de la Facultad Tecnológica de la Universidad Distrital, donde se muestran algunas de las edificaciones donde se podría instalar la unidad externa. 


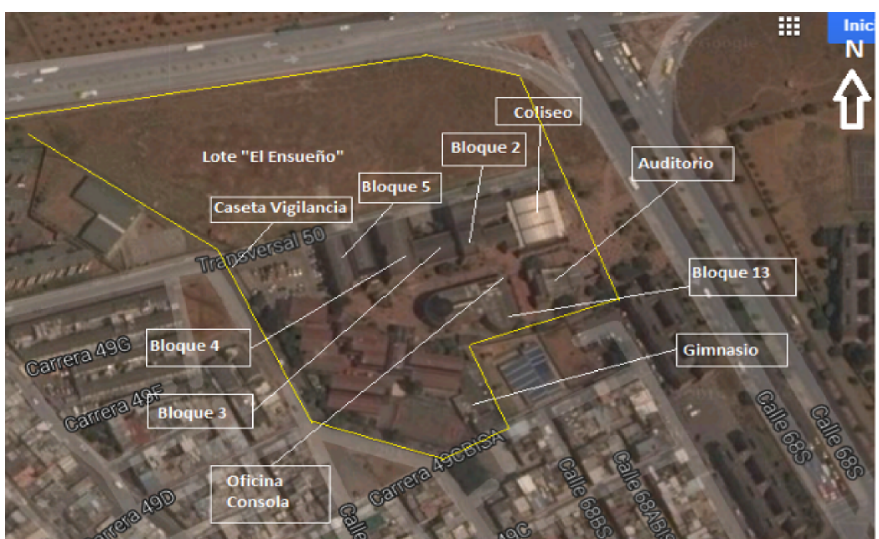

Fig. 4. Fotografía satelital de la Facultad Tecnológica de la Universidad Distrital Francisco José de Caldas. Adaptado de Google Maps. [3]

A. Preselección de posibles sitios para ubicar la unidad externa.

El procedimiento seguido para la selección del sitio de ubicación de la unidad externa se inicia con la identificación y selección de las posibles edificaciones para su instalación, previo a la selección del sitio de ubicación de la consola, el cual corresponde a la oficina del grupo de investigación GIEA.UD, ubicada en el tercer y último piso de del bloque 13, en la parte central de las edificaciones. Según indicaciones de la OMM [4] y del IDEAM se seleccionan edificaciones cuyas cubiertas estuvieran disponibles para una posible instalación, además de contar con las mayores alturas posibles [5] estas corresponden a los tejados o terrazas de la cafetería, los bloques números $1,2,4,5$, el coliseo, el auditorio, el gimnasio, la caseta de vigilancia del parqueadero, y el Lote El Ensueño.

En la Tabla 2 se muestra algunas de las recomendaciones realizadas por el IDEAM, las que se consideran para la selección del lugar más apropiado y ubicar la unidad externa y la unidad de adquisición de datos.

\begin{tabular}{|c|c|}
\hline $\begin{array}{l}\text { IDEAM. CARACTERÍSTICAS TÉCNICAS ESTACIÓN } \\
\text { AUTOMÁTICA METEOROLÓGICA }\end{array}$ & Medidas a tener en cuenta \\
\hline $\begin{array}{l}\text { "La estación debe contar con gabinete hermético } \\
\text { resistente a la corrosión, oxido, lluvia, polvo, y agua para } \\
\text { el alojamiento de la plataforma colectora de datos, } \\
\text { regulador, bateria y así protegerlos ya que serán } \\
\text { instaladas a la intemperie." }\end{array}$ & $\begin{array}{l}\text { Verificar que los materiales de la } \\
\text { estación correspondan a su uso en } \\
\text { exteriores }\end{array}$ \\
\hline $\begin{array}{l}\text { "Pueden incluirse dentro de la garita pilas o alguna } \\
\text { instalación eléctrica de luz fría (neón) para facilitar la } \\
\text { lectura de los instrumentos por la noche." }\end{array}$ & $\begin{array}{l}\text { Instalar un tomacorriente eléctrica } \\
\text { tipo intemperie en caso de requerir } \\
\text { la conexión de equipos o } \\
\text { herramientas eléctricos auxiliares. }\end{array}$ \\
\hline $\begin{array}{l}\text { "De poder elegir el emplazamiento debe hacerse la } \\
\text { elección previendo escasa modificación de las condiciones } \\
\text { actuales en los siguientes } 10 \text { años, por lo menos." }\end{array}$ & $\begin{array}{l}\text { Verificar con las directivas de la } \\
\text { sede que los sitios preseleccionados } \\
\text { en el campus no tendrán } \\
\text { afectaciones estructurales en sus } \\
\text { cubiertas por lo menos en los } \\
\text { próximos } 10 \text { años. }\end{array}$ \\
\hline $\begin{array}{l}\text { "Convendrá que no haya árboles en la parcela reservada } \\
\text { a los instrumentos y de colocarse arbustos en la periferia, } \\
\text { debe dejarse como mínimo una distancia igual o superior } \\
\text { a } 2 \text { metros en torno al pluviómetro y pluviógrafo, } \\
\text { separados éstos por una distancia mínima de } 1,5 \\
\text { metros." "La distancia del pluviógrafo y pluviómetro a } \\
\text { cualquier obstáculo no será inferior a dos veces la altura } \\
\text { de ése obstáculo." }\end{array}$ & $\begin{array}{l}\text { Los sitios preseleccionados deben } \\
\text { estar despejados y no ser afectados } \\
\text { por árboles o sombras de } \\
\text { edificaciones aledañas. }\end{array}$ \\
\hline $\begin{array}{l}\text { "Anemómetros y veletas. Los equipos de sensores de } \\
\text { viento deben captar el viento a una altura normalizada de } \\
10 \text { metros sobre el suelo o terreno efectivo. Es } \\
\text { recomendable que la torre esté bien arriostrada para } \\
\text { evitar movimientos y vibraciones causadas por vientos } \\
\text { fuertes. }\end{array}$ & \\
\hline $\begin{array}{l}\text { "La presencia de antenas o chimeneas voluminosas debe } \\
\text { hacernos escépticos sobre las indicaciones de los } \\
\text { sensores de viento." }\end{array}$ & $\begin{array}{l}\text { Verificar que no existan chimeneas } \\
\text { en próximas al lugar de instalación } \\
\text { de los equipos. }\end{array}$ \\
\hline
\end{tabular}

Tabla 2. Recomendaciones a tener en cuenta a partir de las sugerencias del IDEAM.[5]

\section{B. Criterios para la selección}

Para la selección del sitio de ubicación de la unidad externa de adquisición de datos se consideran aspectos de seguridad y mantenimiento, además de aspectos técnicos.

1. Aspectos de seguridad y mantenimiento.

Se tiene en cuenta:

- Facilidad en acceso para inspecciones de rutina, calibración, mantenimiento y reparación.

- Seguridad y control de acceso a personal autorizado $\mathrm{y}$, dificultad de acceso en situaciones de vandalismo o hurto.

- Visual entre la consola y la unidad externa, supervisión permanente desde la oficina, permitiendo identificar daños u averías a simple vista.

Estar libre de equipos y accesorios en un radio de al menos $2 \mathrm{~m}$ de la unidad exterior.

\section{Aspectos técnicos.}

Se tiene en cuenta:

- Altura mínima de 10 metros sobre el piso, sin obstáculos de edificaciones o arbustos.

- Tipo superficie: que permita la fijación de la unidad externa, que no presente inundaciones y que cumpla las condiciones de seguridad y mantenimiento

- Evaluación de la distancia y obstáculos entre la consola y la unidad externa, minimizando interferencias en la comunicación ocasionada por muros o edificaciones. 
3. Criterios de selección y ponderación.

Después de analizar los diferentes aspectos se consideran seis criterios, que se usan para la selección: Altura, Visual entre consola y unidad externa, Facilidad de acceso, Seguridad (en equipos y personas) Presencia de obstáculos, y Tipo de superficie.

Finalmente para la selección del sitio de ubicación de la unidad externa, se realizó la siguiente ponderación de los criterios, (Tabla 3), dando igual peso a todos ellos y calificándolos entre 0 y 1 , así:

\begin{tabular}{|l|lll|}
\hline Criterio & Ponderación & & \\
\hline Altura & $(>=10 \mathrm{~m})=1 ;$ & $(<=10 \mathrm{~m})=0$ & \\
\hline $\begin{array}{l}\text { Visual Consola- } \\
\text { Unidad.Ext. }\end{array}$ & Directa $=1 ;$ & Indirecta $=0$ & \\
\hline Facilidad de acceso & Alto $=1 ;$ & Medio $=0,5 ;$ & Bajo=0 \\
\hline Seguridad & Alto=1; & Medio=0,5; & Bajo=0 \\
\hline Obstáculos-Arbustos & No=1; & Si=0 & \\
\hline Tipo Superficie & $\begin{array}{l}\text { Plancha concreto=1; } \\
\text { Cúpula }=0,5 ; C \text { Cesped=1 }\end{array}$ & Tejado=0,7; \\
\hline
\end{tabular}

Tabla 3. Criterios de selección y ponderación.

Fuente:. Elaboración propia.

\section{Selección del sitio de ubicación de la unidad externa}

En la Tabla 4 se muestran los criterios de selección con sus respectivas calificaciones asociadas a cada sitio.

\begin{tabular}{|c|c|c|c|c|c|c|c|c|c|c|c|c|c|}
\hline$\stackrel{\circ}{\frac{0}{n}}$ & 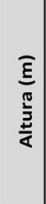 & 鞄 & 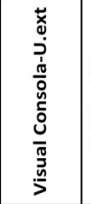 & 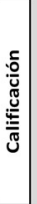 & 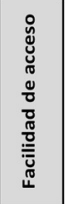 & 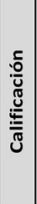 & 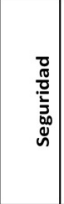 & 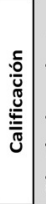 & 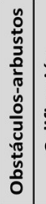 & 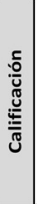 & 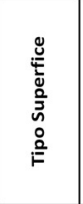 & 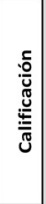 & $\begin{array}{l}\bar{\pi} \\
0 \\
\vdash\end{array}$ \\
\hline Coliseo & 10 & 1 & Directa & 1 & Bajo & 0 & Alta & \begin{tabular}{l|l}
1 & 1 \\
\end{tabular} & No & 1 & Cúpula & 0,5 & 4,5 \\
\hline Auditorio & 10 & 1 & Directa & 1 & Medio & 0,5 & Alta & 11 & No & 1 & $\begin{array}{l}\text { Plancha } \\
\text { concreto }\end{array}$ & 1 & 5,5 \\
\hline Bloque 2 & 12,5 & 1 & Directa & 1 & Medio & 0,5 & Media & \begin{tabular}{l|l}
0,5 & 1 \\
\end{tabular} & No & 1 & Tejado & 0,7 & 4,7 \\
\hline Bloque 3 & 7,5 & 0 & Indirecta & 0 & Medio & 0,5 & Media & 0,5 & $\mathrm{Si}$ & 0 & Tejado & 0,7 & 1,7 \\
\hline Bloque 4 & 12,5 & 1 & Indirecta & 0 & Medio & 0,5 & Media & 0,5 & $\mathrm{Si}$ & 0 & Tejado & 0,7 & 2,7 \\
\hline Bloque 5 & 12,4 & 1 & Indirecta & 0 & Medio & 0,5 & Media & \begin{tabular}{l|l}
0,5 & 1 \\
\end{tabular} & No & 1 & Tejado & 0,7 & 3,7 \\
\hline Caseta Vigilancia & 5,5 & 0 & Indirecta & 0 & Alto & 1 & Media & \begin{tabular}{l|l}
0,5 & 1 \\
\end{tabular} & No & 1 & $\begin{array}{l}\text { Plancha } \\
\text { concreto }\end{array}$ & 1 & 3,5 \\
\hline Lote "El Ensueño" & 0 & 0 & Indirecta & 0 & Alto & 1 & Baja & \begin{tabular}{l|l}
0 & 1 \\
\end{tabular} & No & 1 & Césped & 1 & 3,0 \\
\hline Gimnasio & 2,5 & 0 & Indirecta & 0 & Alto & 1 & Media & \begin{tabular}{l|l}
0,5 & 1 \\
\end{tabular} & No & 1 & $\begin{array}{l}\text { Plancha } \\
\text { concreto }\end{array}$ & 1 & 3,5 \\
\hline
\end{tabular}

Tabla 4. Calificación de criterios de selección del sitio más apropiado para ubicar la unidad externa de adquisición de datos en la Facultad Tecnológica de la Universidad Distrital.

\section{Fuente: Elaboración propia.}

Se observa que el sitio más apropiado es la terraza del Auditorio de la Facultad Tecnológica, con un puntaje de 5,5 puntos sobre 6,0 posibles, seguido por las terrazas del bloque 2 y del Coliseo con puntajes de 4,7 y 4,5, respectivamente. Por lo anterior se decide que el sitio donde se debe instalar la unidad exterior es en la terraza del auditorio Gustavo Caamaño de la Facultad Tecnológica de la Universidad Distrital.

\section{Adecuación del sitio seleccionado.}

Debido a que la terraza del Auditorio no fue diseñada para alojar la unidad exterior de adquisición de datos, es preciso realizar algunas tareas para dejar el sitio apropiado para la instalación de la unidad externa, para lo cual se siguen las recomendaciones planteadas por la OMM, las cuales se presentan en la Tabla 5. En ella se ha adicionado una columna de verificación de cumplimiento de la tarea respectiva.

\begin{tabular}{|l|c|}
\hline \multicolumn{1}{|c|}{ Tarea OMM-258-V-I-ES } & verificación \\
\hline $\begin{array}{l}\text { Preparar sitios de trabajos (zócalos o fundiciones de concreto, montajes, cables } \\
\text { tirantes, protecciones y refugios del equipo); vinculaciones con subcontratistas. }\end{array}$ & $\mathrm{ok}$ \\
\hline $\begin{array}{l}\text { Hacer fabricaciones de taller (escalera) e instalaciones de instrumentos en el campo } \\
\text { y otros sistemas. }\end{array}$ & $\mathrm{ok}$ \\
\hline Realizar pruebas de comunicación entre la consola y unidad externa & $\mathrm{ok}$ \\
\hline Prever acceso para mantenimiento de rutina. & $\mathrm{ok}$ \\
\hline Preparar las instrucciones de operación y mantenimiento, incluyendo correcciones. & Pendiente \\
\hline $\begin{array}{l}\text { Mantener registros de las instalaciones físicas del equipo, modificación, calibración y } \\
\text { reparación. }\end{array}$ & $\mathrm{ok}$ \\
\hline Mantener seguras las prácticas de campo y el taller. & $\mathrm{ok}$ \\
\hline $\begin{array}{l}\text { Diseñar e implementar sitios WWW de Internet e Intranet para proveer servicios } \\
\text { atmosféricos ambientales, información cientifica referente a la atmósfera al equipo } \\
\text { de servicio, la comunidad científica, los asociados y el público en general; }\end{array}$ & Pendiente \\
\hline Inspecciones de rutina de los sitios relevantes; & ok \\
\hline Enrolar y entrenar contratistas, observadores voluntarios. & Pendiente \\
\hline $\begin{array}{l}\text { Suministrar las instrucciones de operación y los procedimientos estándar para } \\
\text { contratistas y observadores; }\end{array}$ & ok \\
\hline $\begin{array}{l}\text { Manejar la entrega de datos de las estaciones de observación para la Oficina Central } \\
\text { con la calidad requerida incluyendo puntualidad; }\end{array}$ & Pendiente \\
\hline Mantener los registros de la estación; & ok \\
\hline
\end{tabular}

Tabla 5. Tareas de adecuación necesarias, sugeridas por la OMM, para poner a punto una estación meteorológica. [4]

\section{RESULTADOS OBTENIDOS}

A la fecha de presentación del presente artículo, algunas tareas sugeridas por la OMM están pendientes de ejecución, y se espera se hayan cumplido cuando el mismo sea publicado. Una vez esto se cumpla, se planea solicitar una visita por parte del IDEAM, con el objetivo de hacer una inspección y lograr la aprobación para ser parte, en un futuro, de la red de estaciones Meteorológicas del IDEAM, con otros centros de investigación, con universidades de Bogotá, y por supuesto con la red PRIDERAS [6] a la cual pertenece y es fundadora la Universidad Distrital.

Se cuenta con una estación meteorológica en la Facultad Tecnológica, la que ha mostrado un buen desempeño en sus primeros meses de funcionamiento, se están registrando datos cada 10 minutos, de las siguientes variables: radiación solar, precipitación, velocidad y dirección del viento, humedad del aire (exterior e interior), temperatura de aire (exterior e interior), presión atmosférica exterior, humedad del suelo, temperatura del suelo, humedad de hoja; adicionalmente se lleva el registro de la carga de las baterías exterior e interior, y de las ráfagas y dirección del viento, para un total de 16 registros. 


\section{A. Ubicación final de los componentes de la estación}

En la figura 5 se observa la proximidad que existe entre el auditorio Gustavo Caamaño, donde se instala finalmente la unidad externa y la oficina donde está instalada la consola receptora y procesadora de datos, la distancia entre los dos dispositivos es algo superior a $10 \mathrm{~m}$, lo que hace propicio para contar con una excelente señal inalámbrica de los datos transmitidos.

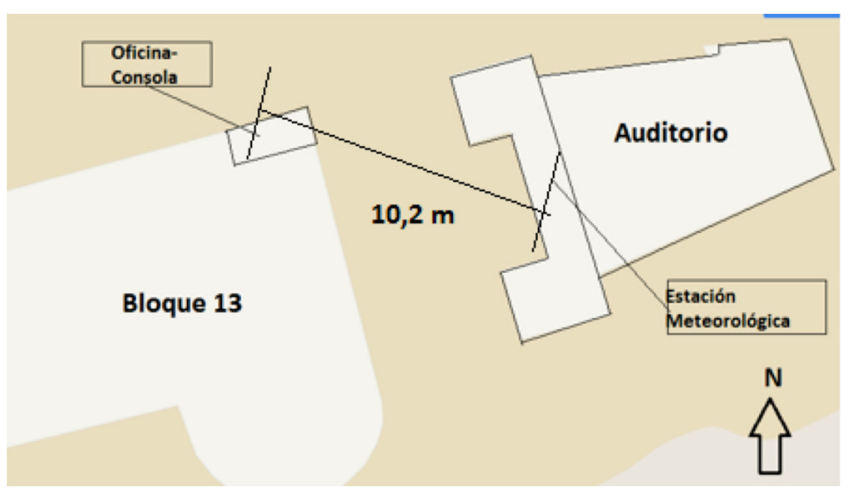

Fig. 5. Plano digital de la ubicación del auditorio y la oficina donde se encuentra la consola. Adaptado de Google Maps [3]

En la Figura 6 se muestran dos fotografías de la unidad exterior, en la foto de la izquierda la unidad externa sobre la terraza del auditorio Gustavo Caamaño, donde se aprecia la adaptación de una puerta y una escalera de acceso; en la foto del lado derecho se muestra el proceso de montaje y calibración de los sensores.
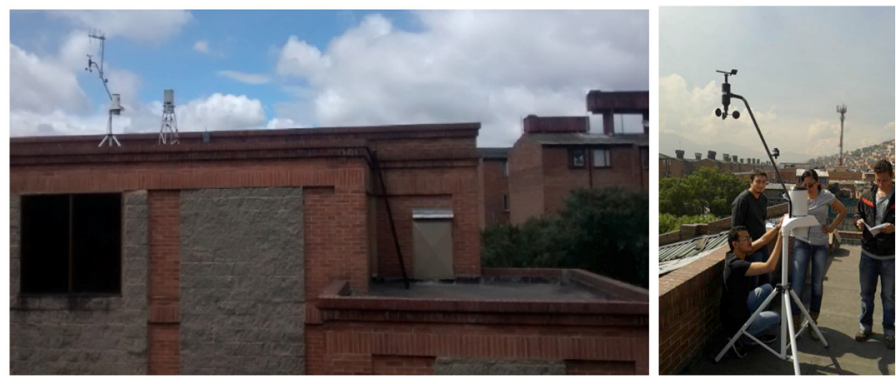

Fig. 6. Fotos de la unidad externa de adquisición de datos en la terraza del Auditorio de la Facultad Tecnológica de la Universidad Distrital. Fuente: Elaboración propia.

\section{B. Resultados preliminares.}

En la Figura 7 se presentan tres gráficas de la radiación solar diaria promedio, donde se muestran los valores máximos, medio y mínimo, en función del tiempo. Allí se indica que la radiación máxima reportada, durante un período de 104 días (del 10 de enero al 23 de abril de 2015), fue de $1271 \mathrm{w} / \mathrm{m}^{2}$, el día 20 de abril de 2015 a las 11:20 am.

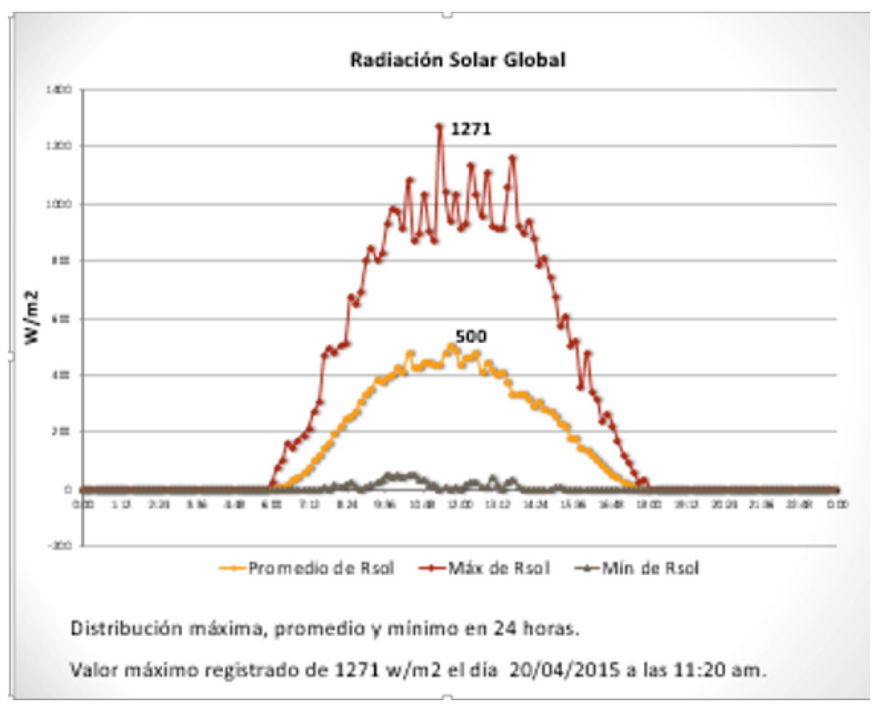

Fig. 7. Distribuciones máxima, promedio y mínima de radiación solar global diaria en $\mathbf{w} / \mathrm{m}^{2}$ durante el período comprendido entre el $10 \mathrm{de}$ enero al 23 de abril de 2015 [7]

En la Figura 8 se muestra el comportamiento de la precipitación promedio diaria, en milímetros, durante el mismo período de 104 días, en ella se puede apreciar que hacia las 20 horas y 10 minutos del día 1 de marzo de 2015 se presenta la máxima precipitación, llegando a un valor de 6,5 $\mathrm{mm}$.

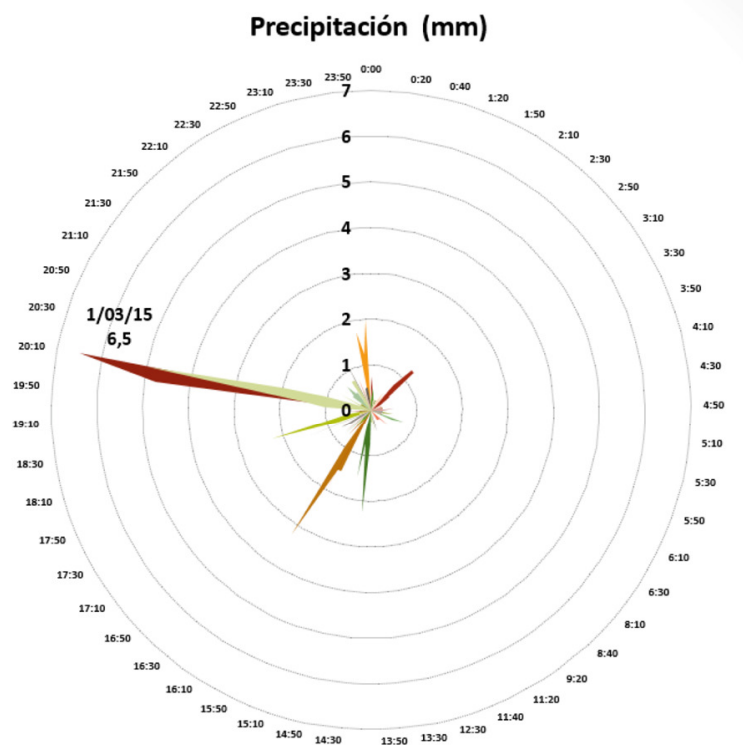

Comportamiento del 10 Enero al 23 de Abril 2015

Fig. 8. Comportamiento de la precipitación promedio en milímetros durante un período de 104 días. Del 10 de enero al 23 de abril de 2015.[7] 


\section{CONCLUSIONES}

La ubicación de la unidad externa y la consola permiten un adecuado registro de los datos, garantizando una transmisión permanente y clara de los mismos, ya que está libre de sombras, de obstáculos físicos y a una distancia relativamente corta, algo más de 10 metros, ideal para esta función.

La terraza del auditorio cumple con las recomendaciones de la OMM y el IDEAM, para ubicar estaciones meteorológicas en espacios urbanos, esto es garantizan seguridad para la estación, y permitir un acceso seguro y controlado a la misma.

El desempeño presentado hasta ahora por la estación, permiten augurar un largo tiempo de funcionamiento adecuado de la misma, situación que vaticina buenas condiciones para poder pertenecer a la red de estaciones del IDEAM, y conformar otras redes con centros de investigación y universidades de la ciudad.

\section{RECOMENDACIONES}

Se debe completar la lista de tareas pendientes como son: el diseño e implementación de un sitio web en Internet e Intranet para proveer la información a la comunidad científica, los asociados y el público en general, enrolar y entrenar personal capacitado para el manejo de la información y realizar el mantenimiento preventivo de la estación, preparar los reportes según normas internacionales, acordar y adoptar, con los demás miembros de las redes proyectadas, el sistema de comunicación de los reportes que se generen.

\section{AGRADECIMIENTOS}

Los autores expresan su agradecimiento a las directivas de la Universidad Distrital Francisco José de Caldas, en especial al Centro de Investigación y Desarrollo Científico (CIDC), por su apoyo económico; a la Facultad Tecnológica por su valiosa colaboración en el proceso de instalación de la estación meteorológica, y al proyecto curricular de Tecnología e Ingeniería Mecánica, por su contribución y aportes realizados.

\section{REFERENCIAS}

[1] E. Portal, «Atlas Cimatológico. primera Parte,» Atlas Climatológico, Mexico, 2009.

[2] TECMES Inteligencia Ambiental, «Manual de Estación Metereológica PEGASUS,» TECMES, Buenos Aires, 2005.

[3] Google Maps, Google Maps, Noviembre de 2014 [En línea]. Disponible en: https://www.google.com.co/maps.

[4] Organización Meteorológica Mundial, «Sistema Mundial
Integrado de Sistemas de Observación de la OMM. Reglamento Técnico,» OMM Naciones Unidas, Ginebra, 2001.

[5] IDEAM, «Manual de referencias para estaciones metereológicas. Características técnicas.,» IDEAM, Bogotá, 2004.

[6] Red PRIDERAS, Red Prideras, Septiembre de 2014. [En línea]. Disponible en: http://www.redprideras.org/.

[7] E. PEGASUS, «Reporte de estación a 24 de abril de 2015,» Universidad Distrital, Bogotá, 2015. 